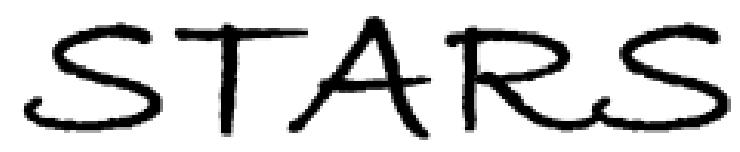

University of Central Florida

STARS

$1-1-2000$

\title{
Microwave response near zero magnetic field in transition-metal- doped silicate glasses
}

\author{
R. R. Rakhimov \\ H. R. Ries \\ D. E. Jones \\ L. B. Glebov \\ University of Central Florida \\ L. N. Glebova \\ University of Central Florida
}

Find similar works at: https://stars.library.ucf.edu/facultybib2000 University of Central Florida Libraries http://library.ucf.edu

This Article is brought to you for free and open access by the Faculty Bibliography at STARS. It has been accepted for inclusion in Faculty Bibliography 2000s by an authorized administrator of STARS. For more information, please contactSTARS@ucf.edu.

\section{Recommended Citation}

Rakhimov, R. R.; Ries, H. R.; Jones, D. E.; Glebov, L. B.; and Glebova, L. N., "Microwave response near zero magnetic field in transition-metal-doped silicate glasses" (2000). Faculty Bibliography 2000s. 2756.

https://stars.library.ucf.edu/facultybib2000/2756

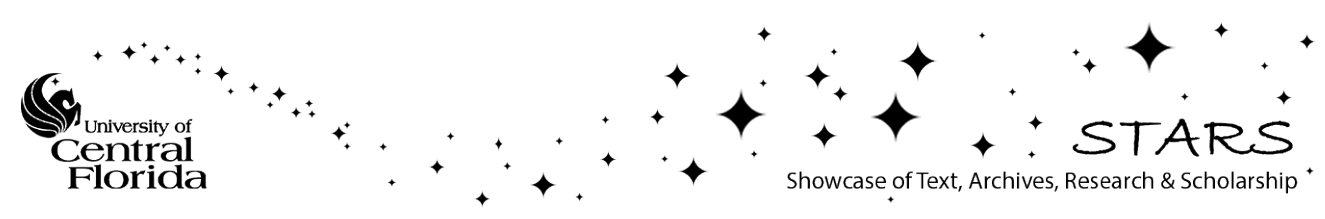




\section{Microwave response near zero magnetic field in transition-metal-doped silicate glasses}

Cite as: Appl. Phys. Lett. 76, 751 (2000); https://doi.org/10.1063/1.125883

Submitted: 07 September 1999 . Accepted: 08 December 1999 . Published Online: 01 February 2000

R. R. Rakhimov, H. R. Ries, D. E. Jones, L. B. Glebov, and L. N. Glebova

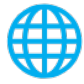

\section{ARTICLES YOU MAY BE INTERESTED IN}

Low-field microwave response and electron paramagnetic resonance identification of valence states of manganese including octahedral $\mathrm{Mn}^{5+}$ in $\mathrm{YAlO}_{3}$ and $\mathrm{CaYAlO}_{4}$

Journal of Applied Physics 95, 5653 (2004); https://doi.org/10.1063/1.1699523

\section{Applied Physics Letters}

Mid-IR and THz frequency combs special collection 


\title{
Microwave response near zero magnetic field in transition-metal-doped silicate glasses
}

\author{
R. R. Rakhimov, ${ }^{\text {a) }}$ H. R. Ries, and D. E. Jones \\ Center for Materials Research, Norfolk State University, Norfolk, Virginia 23504 \\ L. B. Glebov and L. N. Glebova \\ Center for Research and Education in Optics and Lasers, University of Central Florida, Orlando, \\ Florida 32816
}

(Received 7 September 1999; accepted for publication 8 December 1999)

\begin{abstract}
A sharp microwave response centered at zero magnetic field was observed in manganese- and iron-doped $\mathrm{Na}_{2} \mathrm{O}-\mathrm{CaO}-\mathrm{MgO}-\mathrm{SiO}_{2}$ glasses with phase opposite to the normal $\mathrm{Mn}^{+2}$ and $\mathrm{Fe}^{+3}$ paramagnetic signals. The response can be described as magneto-induced microwave conductivity in the dielectric glass that derives from spin-dependent charge migration within the first coordination sphere of the paramagnetic ion. In contrast to the spin-polarized tunneling in ferromagnets between different valence states of metals, the observed effect is due to spin-dependent tunneling that occurs in the vicinity of manganese or iron in diluted paramagnetic systems. (C) 2000 American Institute of Physics. [S0003-6951(00)02706-6]
\end{abstract}

Changes in the electron transport characteristics of materials in varying external magnetic fields are of great fundamental and practical interest. For example, a large magnetoconductance effect (a sharp decrease of electric resistance induced by an applied magnetic field) has been detected in perovskite manganites. This effect is presently explained by enhanced electron hopping migration occurring via a doubleexchange mechanism in mixed valence $\mathrm{Mn}^{+3}-\mathrm{O}-\mathrm{Mn}^{+4}$ structures with an additional magnetic coupling energy that provides ferromagnetism. ${ }^{1-3}$ Microwave magnetoimpedance has been observed in manganites ${ }^{4,5}$ and in amorphous $\mathrm{Fe}-$ $\mathrm{Co}-\mathrm{Si}-\mathrm{B}$ ribbon, ${ }^{6}$ and has been detected by measuring microwave absorption as a function of the applied magnetic field.

In other studies, it was found that the transition to the superconductive state in high-temperature superconductor ceramics leads to nonresonant microwave absorption centered at zero magnetic field. ${ }^{7-10}$ Similar nonresonant microwave absorption signals were detected at low temperatures in borosilicate glasses doped with $\mathrm{Cu}, \mathrm{Ni}, \mathrm{Co}$, and $\mathrm{Fe}$ in high concentrations $(>10 \%){ }^{11}$ The results in Ref. 11 were interpreted in terms of local superconductivity, however, no physical model of the electron mobility was suggested.

In this letter, we report the observation of microwave response near zero magnetic field in the case of diluted paramagnetic systems: $\mathrm{Mn}$ - and Fe-doped insulating $\mathrm{Na}_{2} \mathrm{O}-\mathrm{CaO}-\mathrm{MgO}-\mathrm{SiO}_{2}$ glasses $(\leqslant 1 \%$ doping level). The Mn-doped glasses were studied in more detail than the Fedoped glasses, and we show that the effect is caused by spin-dependent tunneling in the vicinity of the $\mathrm{Mn}^{+2}$ ions.

The glass composition chosen for this work is similar to the standard float glass widely used in the glass industry: (mol. \%) $13 \mathrm{Na}_{2} \mathrm{O}-10 \mathrm{CaO}-6 \mathrm{MgO}-71 \mathrm{SiO}_{2}$. Purified raw materials (with the sum of transition and heavy metal impurities 1-5 ppm) were used to melt high purity glasses by the method described in Ref. 12. All glasses were melted in fused silica crucibles in an electric furnace. Silica and so-

a)Electronic mail: rrakhim@nsu.edu dium, calcium, and magnesium carbonates were used to produce a neutral $\mathrm{Na}_{2} \mathrm{O}-\mathrm{CaO}-\mathrm{MgO}-\mathrm{SiO}_{2}$ glass. To generate oxidizing melting conditions, part of the sodium oxide was introduced as sodium nitrate. To obtain reduced glasses, carbon was added to the batch. High purity undoped glasses, glasses with $\mathrm{MnO}_{2}$ added in amounts ranging from 0.001 to 1 wt. $\%$, and glasses with 1 wt. $\% \mathrm{Fe}_{2} \mathrm{O}_{3}$ were melted in different redox conditions. The glass samples prepared for electron paramagnetic resonance (EPR) studies were in the form of parallelepipeds $2 \times 2 \times 10 \mathrm{~mm}^{3}$ with mass of approximately $0.1 \mathrm{~g}$ each. EPR spectra were taken using a Bruker EMX spectrometer at $9.5 \mathrm{GHz}$ from 77 to $250 \mathrm{~K}$. Polished samples with thickness ranging from 0.015 to 0.5 $\mathrm{cm}$ were prepared for optical absorption measurements, which were made using a Perkin-Elmer 330 double-beam spectrophotometer from 200 to $2500 \mathrm{~nm}$.

No EPR signal exceeding the level of noise was observed in undoped $\mathrm{Na}_{2} \mathrm{O}-\mathrm{CaO}-\mathrm{MgO}-\mathrm{SiO}_{2}$ glasses. The first derivative of microwave absorption versus magnetic field is shown in Fig. 1(a) for glass doped with 0.1 wt. $\% \mathrm{MnO}_{2}$. Microwave absorption spectra of all Mn-doped glasses had a similar response, with magnitude linearly proportional to the manganese concentration. It is known ${ }^{13}$ that manganese in silicate glasses exists in the form of $\mathrm{Mn}^{+3}$ and $\mathrm{Mn}^{+2}$. The $\mathrm{Mn}^{+3}$ ion (electron configuration $3 d^{4}$ ) is not usually detectable by EPR at $9.5 \mathrm{GHz}$. EPR spectra of $\mathrm{Mn}^{+2}$ ions $\left(3 d^{5}\right.$, electron spin $S=5 / 2$ ) in silicate glasses have been known for a long time, ${ }^{14-17}$ and usually contain three sets of lines with effective values of the $g$ factor of approximately $g \approx 4,3$, and 2. Such spectra are explained by a wide distribution of the zero-field splitting parameters. Six hyperfine components due to ${ }^{55} \mathrm{Mn}$ (nuclear spin $I=5 / 2$ ) are usually well observed for the $g \approx 2$ signal. All of these lines with $g \approx 4,3$, and 2 are seen in Fig. 1(a) at magnetic field values of about 1350, 2250 , and $3400 \mathrm{G}$, respectively.

In addition to the well-known $\mathrm{Mn}^{+2}$ EPR spectrum, we have observed a line centered at zero magnetic field [Fig. $1(\mathrm{a})]$ in all $\mathrm{Mn}$-doped $\mathrm{Na}_{2} \mathrm{O}-\mathrm{CaO}-\mathrm{MgO}-\mathrm{SiO}_{2}$ glass samples. We found that this microwave response, as well as the normal EPR signal from $\mathrm{Mn}^{+2}$, is linearly correlated 


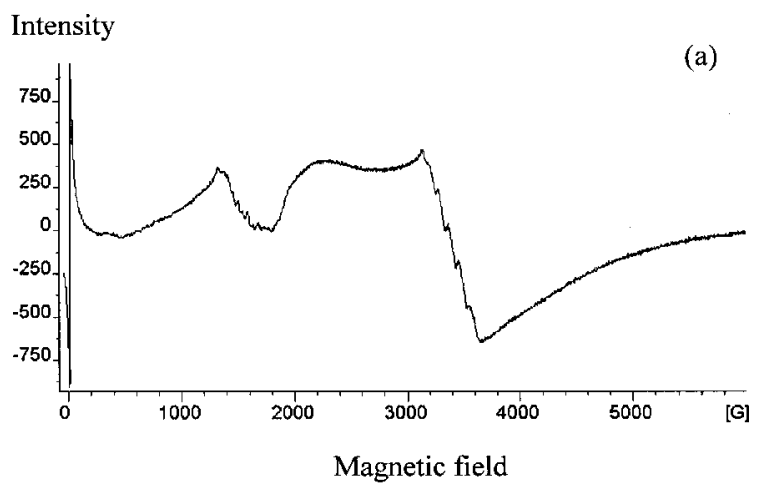

(b)

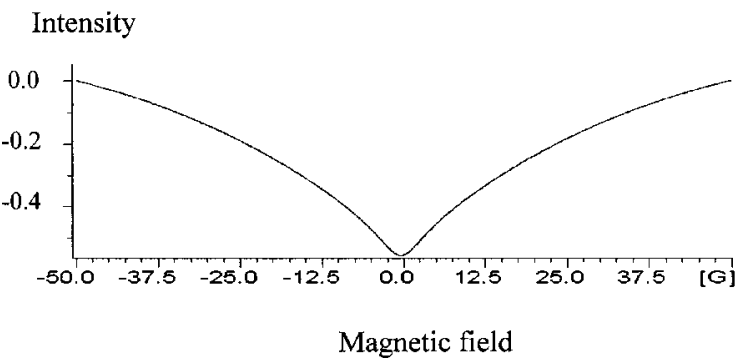

FIG. 1. Microwave absorption (arbitrary units) of the $\mathrm{Na}_{2} \mathrm{O}-\mathrm{CaO}-\mathrm{MgO}-\mathrm{SiO}_{2}$ glass doped with 0.1 wt. $\% \mathrm{MnO}_{2}$ at $77 \mathrm{~K}$. (a) EPR spectrum taken as the first derivative of the microwave absorption. Microwave power applied to the sample was $200 \mathrm{~mW}$. (b) Nonresonant microwave absorption near zero magnetic field.

with the Mn concentration in the glass series. The zero field line, taken as a first derivative of microwave absorption versus magnetic field, has opposite phase relative to the normal EPR absorption due to $\mathrm{Mn}^{+2}$.

There are two possible explanations of the shape of the microwave absorption near zero magnetic field [Fig. 1(b)]. The first possibility is that the microwave absorption exhibits a nonresonant nature, with a minimum value near zero magnetic field that increases to a fixed value as the magnetic field is increased. The alternative explanation would be that there is some resonant paramagnetic absorption in zero magnetic field, but a spectral hole is burned in an inhomogeneous zero field line due to saturation effects. The second explanation is ruled out because the linewidth of about $6 \mathrm{G}$ of the hypothetical burnt hole does not depend on microwave power, and is too small in relation to the manganese hyperfine interaction, which is of the order of $10^{2} \mathrm{G}$.

The nonresonant response with a dip at zero magnetic field was further distinguished from the regular EPR signal with $g \approx 4,3$, and 2 due to the different dependence of the signal magnitude on microwave power and temperature. We could not saturate the nonresonant signal in Mn-doped glasses using applied microwave power up to $200 \mathrm{~mW}$, whereas the saturation of the $g \approx 4,3$, and 2 EPR signals was clearly seen. While the EPR signal in the temperature range from 80 to $250 \mathrm{~K}$ decreases by $30 \%$, the nonresonant signal drops by four times. Thus, the low field response should not be described as the normal EPR signal from $\mathrm{Mn}^{+2}$ or $\mathrm{Mn}^{+3}$ ions, despite the linear correlation with the Mn concentration in the glass series mentioned previously.

To distinguish which valence state of $\mathrm{Mn}$ is responsible for the zero-field microwave response, glasses with $0.1 \%$ of

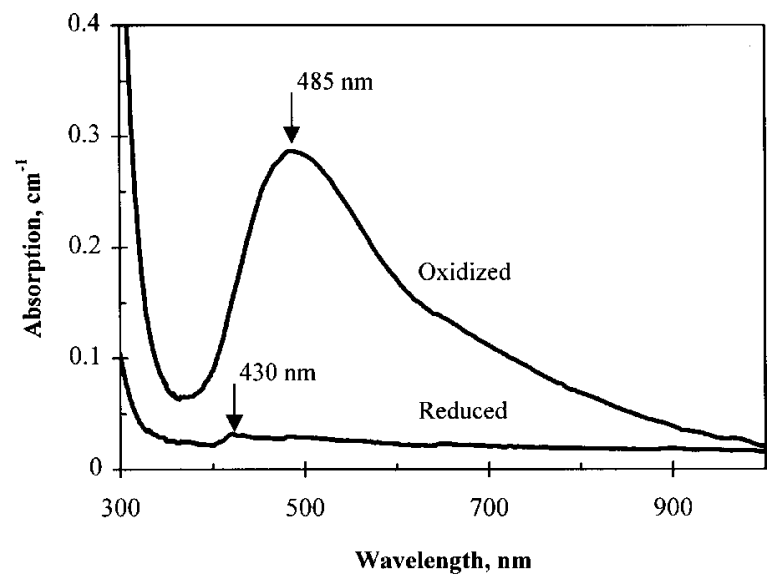

FIG. 2. Optical absorption spectra of the $\mathrm{Na}_{2} \mathrm{O}-\mathrm{CaO}-\mathrm{MgO}-\mathrm{SiO}_{2}$ glasses doped with 0.1 wt. $\% \mathrm{MnO}_{2}$ and synthesized in oxidized and reduced atmosphere.

$\mathrm{MnO}_{2}$ were synthesized in strong reducing and oxidizing conditions. In oxidized conditions, the optical absorption spectra of glasses synthesized show the presence of $\mathrm{Mn}^{+3}$ ions with absorption band ${ }^{13}$ having maximum at $\lambda$ $=485 \mathrm{~nm}$ (Fig. 2). Although the concentration of $\mathrm{Mn}^{+2}$ is significantly higher than the concentration of $\mathrm{Mn}^{+3}$ even in oxidized glass, ${ }^{13}$ the specific absorption of $\mathrm{Mn}^{+2}$ ions is significantly lower ${ }^{14}$ and therefore the optical absorption of $\mathrm{Mn}^{+2}$ cannot be detected on the strong background of the $\mathrm{Mn}^{+3}$ absorption. When we used strong reducing conditions for glass melting, we observed almost complete suppression of the absorption band due to $\mathrm{Mn}^{+3}$, but found a small absorption band near $430 \mathrm{~nm}$ (Fig. 2) which was attributed to $\mathrm{Mn}^{+2} .{ }^{13}$ Thus, we determined from the optical data that the reducing conditions increased the concentration of $\mathrm{Mn}^{+2}$ relative to the concentration of $\mathrm{Mn}^{+3}$ in glass with the same total concentration of manganese $(0.1 \%)$. We then found that the intensity of the normal EPR signal due to $\mathrm{Mn}^{+2}$ and the low field microwave response both increased by the same value in the reduced glass compared to the oxidized glass. Thus, we can conclude that $\mathrm{Mn}^{+2}$ ions are responsible for the line near zero magnetic field, although this line cannot be described as a paramagnetic absorption of the $\mathrm{Mn}^{+2}$ ion.

Since the EPR spectrum is taken as the first derivative of the absorption spectrum, the reversed phase of the nonresonant line means that the microwave absorption has a minimum at zero magnetic field, and it increases with the applied magnetic field $H$ and reaches some plateau value. Microwave absorption in glasses derives from dielectric losses due to electric charge displacement. ${ }^{18}$ The time-averaged power $W$, dissipated in a system carrying a current density $j$ in an alternating electric field $E=E_{0} \exp (-i \omega t)$ is given by (see, for example, Ref. 19, p. 225)

$$
W=j E=(1 / 2) \epsilon_{0} \operatorname{Im}(\epsilon) \omega E_{0}^{2},
$$

where $\epsilon_{0}$ is the permittivity of vacuum and $\operatorname{Im}(\epsilon)$ is the imaginary part of the dielectric constant $\epsilon$ which depends upon the microwave frequency $\omega$. In a dielectric, the only current which can flow is the Maxwell displacement current $j=d\left(\epsilon \epsilon_{0} E\right) / d t$ induced by microwave electric field $E$. Since $j=\sigma E$, where

$$
\sigma=(1 / 2) \epsilon_{0} \operatorname{Im}(\epsilon) \omega
$$


is the microwave conductivity, we shall further describe magnetic field dependent microwave absorption in the glass, $W=\sigma E_{0}^{2}$ in terms of changes in microwave conductivity $\sigma(H)$ with the applied static magnetic field $H$. Experimentally observed low field microwave response is

$$
\Delta W=W(H)-W(0)=\Delta \sigma E_{0}^{2},
$$

where $\Delta \sigma=\sigma(H)-\sigma(0)$ is the change in microwave conductivity induced by the magnetic field $H$. Since dielectric loss in the absence of static magnetic field is given by $W(0)=\sigma(0) E_{0}^{2}$, the observed response can be presented as

$$
\Delta W=[\Delta \sigma / \sigma(0)] W(0) .
$$

The term $\Delta \sigma / \sigma(0)$ describes relative changes in microwave conductivity induced by the external magnetic field, that is, magnetoconductance.

The explanation of magnetoresistance in perovskite manganites ${ }^{3}$ relies on assumptions about ferromagnetic or exchange coupling between manganese ions with different charge states. Such assumptions are not justified in the present case of nonresonant absorption in glasses observed with concentrations of $\mathrm{Mn}$ as low as $0.001 \%$. Therefore, the observed effect cannot be related to changes in d.c. conductivity of the glass, but should be considered as the enhancement of dielectric loss by the external magnetic field, or microwave magnetoconductance $\Delta \sigma / \sigma(0)$.

We propose the following model for the microwave magnetoconductance induced by $\mathrm{Mn}^{+2}$ in the glass. First, we assume that the octahedral coordination sphere of the $\mathrm{Mn}^{+2}$ ion is characterized by a nonzero electric dipole moment due to partially covalent bonds between manganese and oxygen atoms $\mathrm{Mn}^{+2-\delta}-\mathrm{O}^{-2+\delta}$. If the charge $+\delta$ did not exist or were equally distributed between all oxygen atoms, there would be no electric dipole moment. One of the five unpaired electrons from the $d$ shell of $\mathrm{Mn}^{+2}$ will pair with one of the two electrons from the $p$ orbital of oxygen to provide covalent bonding, so that one unpaired electron spin will remain on the oxygen atom. Alternatively, in the general case, the electron spin density will be partially delocalized to the oxygen atom. In either case, the total electron spin of the system, $S=5 / 2$, remains unchanged. Next, we suppose that the bonding of $\mathrm{Mn}^{+2-\delta}-\mathrm{O}^{-2+\delta}$ may switch to other oxygen atoms in the vicinity of $\mathrm{Mn}^{+2}$,

$$
\mathrm{O}^{-2} \ldots \mathrm{Mn}^{+2-\delta}-\mathrm{O}^{-2+\delta} \rightarrow \mathrm{O}^{-2+\delta}-\mathrm{Mn}^{+2-\delta} \ldots \mathrm{O}^{-2} \text {. }
$$

In the absence of an external magnetic field $H$, the spontaneous migration of the charge $+\delta$ between oxygen atoms in the octahedron is the source of the dielectric loss $W(0)$.

When an external magnetic field is applied, $H \neq 0$, it will reduce the fluctuation of unpaired electron spins and align them in one direction, so that an electron can tunnel from one oxygen atom to another without changing the total spin of the system,

$$
\begin{aligned}
\mathrm{O}^{-2}(\uparrow \downarrow) \ldots \mathrm{Mn}^{+2-\delta}(\uparrow \uparrow \uparrow \uparrow \ldots \uparrow \downarrow \ldots \uparrow) \mathrm{O}^{-2+\delta} \\
\quad \rightarrow \mathrm{O}^{-2+\delta}(\uparrow \ldots \uparrow \downarrow \ldots \uparrow \uparrow \uparrow \uparrow) \mathrm{Mn}^{+2-\delta} \ldots \mathrm{O}^{2}(\uparrow \downarrow) .
\end{aligned}
$$

This results in an increase of the dielectric loss up to the value of $W(H)$ due to the additional tunneling migration of the charge $+\delta$.
A low field response similar to that observed in the Mndoped glass series was detected in the case of the Fe-doped $\mathrm{Na}_{2} \mathrm{O}-\mathrm{CaO}-\mathrm{MgO}-\mathrm{SiO}_{2}$ glass, in addition to the well-known EPR signal of $\mathrm{Fe}^{+3}, S=5 / 2$ with absorption maximum near $g \approx 4 .{ }^{16}$ Detailed analysis of the low field microwave response in Fe-doped silicate glasses will be published elsewhere. However, this result shows that magneto-induced nonresonant microwave absorption is not a unique feature of manganese and the search for new microwave magnetoconductors should be continued.

In summary, we have observed magneto-induced nonresonant microwave absorption caused by paramagnetic ions in insulating silicate glass. This phenomenon is described in terms of spin-dependent dielectric loss or microwave magnetoconductance that derives from the tunneling charge migration between oxygen atoms via a paramagnetic dopant ion. The spin-dependent tunneling described in the present work results in a distinct, detectable response in the low magnetic field region, which makes transition-metal-doped glasses potential magnetosensitive materials. The value of microwave magnetoconductance $\Delta \sigma / \sigma$ will define the potential usefulness of this type of glasses for magnetoelectronics. Therefore, more detailed studies should be undertaken to measure the absolute values of magneto-induced microwave losses in these glasses.

The financial support for this research was provided by the National Science Foundation (HRD-9805059) and Visteon Glass System.

${ }^{1}$ R. von Helmholt, J. Wecker, B. Holzapfel, L. Schultz, and K. Samwer, Phys. Rev. Lett. 71, 2331 (1993).

${ }^{2}$ K. Chahara, T. Ohno, M. Kasai, and Y. Kozono, Appl. Phys. Lett. 63, 1990 (1993)

${ }^{3}$ H. Y. Hwang, S.-W. Cheong, N. P. Ong, and B. Batlogg, Phys. Rev. Lett. 77, 2041 (1996).

${ }^{4}$ S. E. Lofland, S. M. Bhagat, S. D. Tyagi, Y. M. Mukovskii, S. G. Karabashev, and A. M. Balbashov, J. Appl. Phys. 80, 3592 (1996).

${ }^{5}$ V. V. Srinivasu, S. E. Lofland, and S. M. Bhagat, J. Appl. Phys. 83, 2866 (1998).

${ }^{6}$ A. N. Medina, M. Knobel, S. Salem-Sugui, and F. G. Gandra, J. Appl. Phys. 79, 5462 (1996).

${ }^{7}$ A. Gould, S. M. Bhagat, F. C. Wellstood, and S. Tyagi, Solid State Commun. 81, 339 (1992).

${ }^{8}$ J. S. Ramachandran, M. X. Huang, S. M. Bhagat, K. Kish, and S. Tyagi, Physica C 202, 151 (1992).

${ }^{9}$ J. S. Ramachandran, M. X. Huang, and S. M. Bhagat, Physica C 234, 173 (1994).

${ }^{10}$ V. Seshu Bai, P. V. Patanjali, and S. M. Bhagat, J. Supercond. 8, 299 (1995).

${ }^{11}$ A. I. Veinger, A. G. Zabrodsky, T. V. Tisnek, L. B. Glebov, K. O. Shchavelev, and O. S. Shchavelev, Physica C 235-240, 2064 (1994).

${ }^{12}$ L. B. Glebov, L. B. Popova, M. N. Tolstoi, and V. V. Rusan, Sov. J. Glass Phys. Chem. 2, 547 (1976).

${ }^{13}$ W. A. Weyl, Colored Glasses (The Society of Glass Technology, Sheffield, 1951).

${ }^{14}$ R. H. Sands, Phys. Rev. 99, 1222 (1955).

${ }^{15}$ D. L. Griscom and R. E. Griscom, J. Chem. Phys. 47, 2711 (1967).

${ }^{16}$ D. L. Griscom, J. Non-Cryst. Solids 40, 211 (1980).

${ }^{17}$ P. Rumori, B. Deroide, N. Abidi, H. El Mkami, and J. V. Zanchetta, J. Phys. Chem. Solids 59, 959 (1998).

${ }^{18}$ J. Wang and C. A. Angell, Glass Structure by Spectroscopy (Marcel Dekker, New York, Basel, 1976).

${ }^{19}$ H. M. Rosenberg, The Solid State (Oxford Science, Oxford, 1988). 